Nummern zu erkundigen. Gegen einen Erlag von $6^{1 / 2} \mathrm{kr}$. CM. pr. Quartal bei dem betreffenden Postamte wird das Blatt auch in die Wohnung gestellt.

Alle Buchhandlungen und Postämter des In- und Auslandes nehmen ebenfalls Pränumerationen an.

In s e r a t e werden mit $5 \mathrm{kr}$. CM. für die ganze Petitzeile berechnet.

Von den vier ersten Jahrgängen des botanischen Wochenblattes sind noch vollständige Exemplare zu haben und können durch alle Buchhandlungen un nachfolgenden Preis bezogen werden:

I., II. und III. Jahrgang $\ldots \ldots \ldots \ldots \ldots \ldots \ldots$ à a fl. CM. IV. Jahrgang ...............

\title{
Die Redaction.
}

(Wieden, Neumannsgasse Nr. 331.)

\section{Eine Flora von Süd-Tirol von Dr. Facchin i.}

Von Fr. Hausmann.

Der Professor der Naturgeschichte am hiesigen Ober-Gymnasium Pater Vincenz G r e d l e r machte im verflossenen Sommer auf einem seiner wissenschaftlichen Ausflüge in die Thäler von Fassa, Gröden und Enneberg einen köstlichen literarischen Fund. Es ist diess das hinterlassene vollständige Manuscript einer Flora des südlichen Tirols von Dr. F a c chini, und dem gefälligen Entgegenkommen des Hrn. Professors, meines Freundes, verdanke ich die unbeschränkte Verfügung über dasselbe. Dr. F a c ch i n i hat sich auf seinen vieljährigen Excursionen über gar manche unserer Alpenpflanzen ein selbstständiges, von dem der meisten übrigen Botaniker abweichendes Urtheil gebildet, und selbes in erwähntem Manuscripte zu begründen gesucht. Auf dass nun diese Ansichten und-Erfahrungen des Verstorbenen unvermischt und ungewässert den Freunden unserer Alpenflora zugänglich werden, gedenke ich nächstens in irgend einer botanischen Zeitschrift das bezügliche Manuscript zu veröffentlichen, und glaube dadurch des Dankes aller deutschen Botaniker versichert zu sein, so wie das Andenken des Verblichenen am besten zu ehren. Das Manuscript ist in einem zierlichen Latein, und einer kräftigen, oft etwas derben Sprache, wie man sie im Umgange mit F a c chin i gewohnt war, abgefasst. Was die Umgränzung der Arten anbelangt, so zeigt sich eine starke Hinneigung $\mathrm{zu}$ den Zeiten Linne's und Willdenow's, und die Bovorworlung der Zusammenziehung einer grossen Anzahl von Arten, namentlich der Gattungen Hieracium, Viola, Rosa, Polygala, Orobanche etc. dürfte kaum auf den Beifall des grösseren Theiles unserer Botaniker zu zählen haben, so wie ich glaube, dass die Aufgabe einer Specialflora mehr die Erforschung und Feststellung aller Pflanzenformen irgend eines Florengebietes, als die Frage was Art, was nicht Art, ist. Glücklicherweise hat diese Abneigung F a c chin i's gegen die meisten jener Arten, die ihre Entstehung der neuern Zeit verdan- 
ken, ihn doch nicht vermocht, uns die Beschreibung einiger neuer, von ihm in Süd-Tirol aufgefunderrer Pflanzen vorzuenthalten, wenn er gleich, wie aus dem Contexte des Manuscriptes hervorgeht, bei ein paar derselben nur beziehungsweise über ihre Artengüte im Li in ne'schen Sinne mit sich selbst im Reinen war. Um so mehr muss ich bedauern, dass es mir bisher nicht gelungen ist, Exemplare der drei folgenden $\mathrm{F}$ a c ch in i'schen Arten, deren allsogleiche Veröffentlichung ich nicht verschieben zu sollen glaube, zu erhalten. Ich gebe die Beschreibung wortgetreu, nur habe ich der Kürze wegen nicht alle einzelnen Standorte angeführt, so wie ich F acch in i's eigene weilläufigere Bemerkungen zu diesen seinen Arten zuın späteren Abdrucke vorbehalte.

Sempervivum dolomiticum Facchini.

Sempervivum: propaginum foliis brevissime ciliatis, corolla stellata, petalis lanceolatis. Caetera Sempervivi Funkii. - Crescit in alpium regione inferiore, media, et suprema ad extrema juga usque, in alpe Seekofl (Pusterthal), in monte di Pozza (Fassa) etc. etc., solo calcareo-dolomitico puro, unde nomen.

\section{Festuca brennia Facchini.}

Festuca: paniculat ramis semiverticillatis, spiculis multifloris, foliis culmeis planis.

A F. pilosa, cui similis, differt, praeter allatas notas, statura majore, culmis sesquipedalibus et altioribus, quamvis locis frigidis et sterilibus crescat. Spiculae 6-8 florae. - In monte Schneeberg in valle: Passeier, in alpe Giumella (Fassa) etc. etc. a regione suprema culturae, solo granitico $(18 \$ 4$ et $184 \mathrm{i})$.

A Brennis populis dicta, qui monti: Brenner nomen dedisse creduntur.

Epilobium salicifolium Facchini.

Ep.: stoloniferum, foliis oblongo-lanceolatis, stigmate quadrifido. $E$. origanifolio simile, a quo differt foliis angustioribus et stigmate diviso.

Ad oras piscinae sub monte Schleern occidentem versus.

Bemerken muss ich hier noch, dass die von A mbrosi im botanischen Wochenblatte 1853 , $\mathrm{Nr}$. 47 und 50 , nach dem $\mathrm{F}$ a cc h in i'schen Herbare veröffentlichte Daphne rupestris $\mathrm{F}$ a c ch. und Carex reclinata $\mathrm{Facch}$., wie nicht nur aus dem Wortlaute der Beschreibung, sondern auch von Exemplaren hervorgeht, die ich später als von A mbrosi selbst herrührend, einzusehen Gelegenheit halte, identisch sind mit Daphne petraea Leybold und Carex ornithopodioides $\mathrm{H}$ a u $\mathrm{s}$ a $\mathrm{n} n$ (Flora 1853, Nr. 6 und 15), und daher an Priorität den letzteren nachstehen müssen, was A mbros i in seiner bald zu gewärtigenden Flora des italienischen Tirols sicherlich auch anerkennen wird.

B o tze n, den 1. December 1854. 